\title{
Self-Interacting Right-Handed Neutrinos as Warm Dark Matter and Small-Scale Cosmology "Crisis"
}

\author{
Nick E. Mavromatos ${ }^{* \dagger}$ \\ King's College London, Physics Department, Theoretical Particle Physics and Cosmology \\ Group, Strand, London WC2R 2LS, UK \\ E-mail: Nikolaos.Mavromatos@kcl.ac.uk
}

In the talk, I review some recent developments on the topic of self-interacting Dark Matter (SIDM), paying particular attention to restrictions on the SIDM (total) cross section from the use of novel observables in merging galactic structures, as well as the rôle of SIDM on the milky way halo and its central region. In this latter context, I discuss interesting particle-physics inspired SIDM models involving self-interacting right-handed (Majorana) neutrino DM (with mass of a few tens of $\mathrm{keV}$ ), that may exist in minimal extensions of the Standard Model. I also describe briefly the phenomenology of these models, and argue that they can tackle the basic problems of the "small-scale Cosmology crisis".

Corfu Summer Institute 2016 "School and Workshops on Elementary Particle Physics and Gravity" 31 August - 23 September, 2016

Corfu, Greece

\footnotetext{
* Speaker.

${ }^{\dagger}$ This work is supported in part by STFC (UK) under the research grant ST/L000326/1.
} 


\section{Introduction}

Although the simple Friedmann-Lemaître-Robertson-Walker (FLRW) cosmological model for a homogeneous and isotropic Universe works very well in describing several evolutionary aspects of it, nevertheless about $\sim 90 \%$ of its total energy budget still remains a mystery. Only $5 \%$ of the energy budget of the Cosmos, consisting of ordinary matter and radiation (that is mainly protons, neutrons, electrons, as well as photons), is well understood [1]. Unknown forms of Matter (Dark Matter (DM) - 27\%) and Vacuum Energy (Dark Energy- 71\%) have to be included in order to explain the plethora of cosmological observations. At large scales the current data are explained well by the $\Lambda \mathrm{C}$ (old)DM framework.

In this talk, we shall concentrate on the self interaction (SI) aspects of dark matter (DM) [2]. From a particle physics point of view, DM has many candidates from physics beyond the Standard Model (SM), such as supersymmetric partners, axions, sterile neutrinos, etc. In fact the current cosmological data from Planck satellite [3], and its predecessor WMAP [4], on the amount of allowed dark matter abundance imply stringent constraints on collider searches for new particles, e.g. those implied by supersymmetry (SUSY). From an astrophysical point of view, DM is viewed simply as the "missing" amount of matter in the Universe as compared with expectations from standard FLRW cosmology. It has been studied mostly in a model independent way, by performing N-body simulations of non-interacting Newtonian massive bodies. Such simulations aimed at reproducing the large scale structure of the (observable) universe, and are intimately linked with (though do not depend explicitly on) the particle mass range, nature, and physics of the particle decoupling from the primordial plasma. These properties lead to a characteristic free-streaming length of the dark candidate at the time of formation of the gravitationally bounded DM seeds. Typically, weakly interacting cold massive leptons, with masses of order $\sim 100 \mathrm{GeV}$, decouple at non-relativistic energy scales, implying a very small free-streaming length, far below the scale of dwarf galaxies, with the corresponding 'Jeans' mass of order $10^{-4} M_{\odot}$ [5]. The lower bound on the mass of such dark matter is associated with a cutoff in the matter power spectrum at the lowest end of the free-streaming length (i.e. largest wavelength number $k$ ), which is supplemented by the effect of acoustic oscillations owing to the coupling of the radiation field with the CDM particles [5]. On the contrary, warmer (i.e. of keV mass) DM particles that decouple while still relativistic, can become non-relativistic already at the radiation era, with free-streaming scales typically of a galaxy size, wiping out all possible structure below such a scale. Consequently, this will affect the building-up of DM halos (and the galaxies within), by lowering the inner halo mass concentration and by suppressing the number of small satellite halos [6]. In this way, re-ionization studies based on N-body simulations of the Universe at redshifts $z \sim 20$ [7], when combined with the evidence from the WMAP observations [4], exclude any Warm DM (WDM) with mass less than $10 \mathrm{keV}$ as being the dominant DM component in the Universe (thereby excluding light gravitino models of DM with $m_{X} \simeq 5 \mathrm{keV}$ ). This is so because the suppression of formation of such low mass objects in this early epoch makes the formation of primordial molecular hydrogen gas clouds very inefficient, and thus inconsistent with the large optical depth observed by the WMAP satellite [7]. Complementary constraints, confirming such exclusion regions, are those due to observations on the number of Milky Way satellites, which is about an order of magnitude greater than that predicted by WDM numerical simulations, [8] and the Ly- $\alpha$ forest constraints [9]. 
It should be noted at this stage that such structure formation arguments can only place a lower bound on the mass of the WDM candidate. The reader should bear in mind that Warm Dark Matter with masses $m_{X} \gtrsim 100 \mathrm{keV}$ becomes indistinguishable from Cold Dark Matter (CDM), as far as large-scale structure formation is concerned [6]. Hot dark matter (due to the three active lefthanded neutrino species of the SM) is also excluded as the dominant source of DM in the universe by the upper limits for the corresponding contributions $\Omega_{v}$ to the Universe energy density, which according to the recent Planck 2015 data [3], combined with Lensing, Baryon Acoustic oscillation and Lyman $\alpha$ data, are bounded from above as follows: $\Omega_{v} h^{2}=\frac{\sum_{i=1}^{3} m_{i}}{94.0 \mathrm{ev}} \leq 0.0025$. in a standard notation, where the energy densities are expressed in units of the critical density of the universe, and $m_{i}$ denote the light neutrino masses. From this, one also obtains a cosmological bound on the sum of the masses of the three light neutrino species $\sum_{i=1}^{3} m_{i} \leq 0.23 \mathrm{eV}$.

The exclusion of HDM and (few keV) WDM from being the dominant source of DM at the large scale Universe has prompted a plethora of works, both in particle physics and astrophysics, on the CDM model, which depending on its thermal history may be constrained significantly not only by astrophysical observations but also at particle colliders, such as the Large Hadron Collider (LHC) at CERN, assuming that DM is particle in origin [10]. One of the main reasons for the particle physicists taking a great interest in the DM problem was the so called Weakly Interacting Massive Particle (WIMP) coincidence or 'miracle' [11]. This is associated with the fact that the observed relic abundance of DM in the universe [3], $\Omega_{\chi} \sim 0.22$ for a neutral particle of mass $m_{\chi}$, assumed to be thermal ${ }^{1}$ having a freeze-out temperature [10] $k_{B} T \simeq m_{\chi} / 20$ (in natural units, with $k_{B}$ the Boltzmann constant), which is either stable or it has at least a proper life time longer than the age of the Universe, can be achieved by particles with annihilation cross sections to SM particles $\sigma(\chi \chi \rightarrow \mathrm{SM})$ of order of those of the weak interactions

$$
\left.\Omega_{\chi} \simeq \frac{0.1 \mathrm{pb} \cdot \mathrm{c}}{\langle\sigma(\chi \chi \rightarrow \mathrm{SM} v\rangle} \simeq 0.22 \text { for } \sigma(\chi \chi \rightarrow \mathrm{SM} v\rangle\right) \simeq 3 \cdot 10^{-26} \mathrm{~cm}^{3} \mathrm{~s}^{-1}
$$

with $v$ the relative velocity. A plethora of modern particle physics models, with DM masses ranging from a few hundreds of $\mathrm{GeV}$ to a few $\mathrm{TeV}$, especially supersymmetric ones, have been tested against cosmological data at current colliders, such as the LHC, using (1.1), with no evidence for the existence of such particles. This may not come as a surprise. DM may not be thermal, e.g. it may be due to axions or other non thermal particles, such as sterile neutrinos, and in general one may even face a situation where more than one dominant dark matter species exist in the universe. In the latter case, some of the stringent constraints obtained by comparing collider data to cosmology may be relaxed [10].

In fact the $\Lambda \mathrm{CDM}$ paradigm for DM, on which the aforementioned WIMP miracle is based, in spite of offering a convincing explanation for the observed data [3], namely Cosmic Microwave Background (CMB), Baryon Acoustic Oscillations (BAO), weak and strong lensing data, nevertheless it fails to account for observations at smaller scales. In particular, at galaxy scales, there are serious unresolved problems, related mainly to discrepancies in the distribution of matter in the inner halo regions of galaxies between numerical simulations based on $\Lambda \mathrm{CDM}$ and observations. These issues present serious challenges to the $\Lambda \mathrm{CDM}$ paradigm that call urgently for explanations.

\footnotetext{
${ }^{1}$ Although, it should be noted that non-thermal WIMP Miracle models do exist in the literature [12].
} 
Various proposals for a resolution of these problems have been proposed, some of which go beyond the $\Lambda$ CDM paradigm, including either self interactions among the dark matter particles [13,14, 15], due to novel kind of forces exclusive to the dark sector, or studying viscous properties of DM [16], which have important back reaction effects on the standard cosmological equations.

In this review we shall concentrate on SIDM models and their galactic phenomenology. The structure of the article is the following: in the next section 2, we outline the problems/challenges faced by the simulations based on the $\Lambda \mathrm{CDM}$ model as regards the observed distribution of matter at galactic scales and discuss potential resolutions, among which self interactions of DM, which we place our emphasis on. In section 3, we present a particle physics model of self-interacting WDM provided by right-handed Majorana neutrinos, which addresses successfully these challenges in a way consistent with observations. The model is also consistent with the rest of the cosmological and particle physics data for right handed neutrinos. Finally, in section 4, we present our conclusions.

\section{Small-Scale Cosmology Crisis and Self-interacting Dark Matter}

There are three major problems/challenges to the $\Lambda \mathrm{CDM}$ model at galactic scales (which we can collectively call "small-scale cosmology crisis or problems"), which we shall identify here:

(i) The Core-Cusp problem (or, as is also known, the cuspy-halo problem), refers to a discrepancy between the observed dark matter density profiles of low-mass galaxies and the density profiles predicted by cosmological N-body simulations. Characteristically, all the $\Lambda$ CDM-based (DM only) simulations form dark matter halos which have "cuspy" dark matter distributions, with the density increasing steeply, i.e. as $\rho \propto r^{-1}$, at small radii. This is, e.g., evidenced in the standard NavarroFrenk-White (NFW) DM profile [17]. On the contrary, the rotation curves of most of the observed dwarf galaxies indicate flat central density profiles ("cores") [18].

(ii) The "missing satellite problem" (or, as is also known, the dwarf galaxy problem), arises from a discrepancy between $\Lambda \mathrm{CDM}$-based numerical cosmological simulations that predict the evolution of the distribution of matter in the universe - pointing towards a hierarchical clustering of DM (where smaller halos merge to form larger halos) - and observations. Although there seem to be enough observed normal-sized galaxies to account for such a numerical distribution, the number of dwarf galaxies is orders of magnitude lower than that expected from the simulations. As a concrete example, we mention that there were observed to be around 38 dwarf galaxies in the Local Group, and only around 11 orbiting the Milky Way, yet one dark matter simulation predicted around 500 Milky Way dwarf satellites [19].

(iii) The too big to fail problem, that is a discrepancy arising between the most massive subhaloes predicted in (dissipationless) $\Lambda \mathrm{CDM}$ simulations and the observed dynamics of the brightest dwarf spheroidal (dSph) galaxies in the Milky way (see Fig. 1). In other words, the $\Lambda$ CDM simulations predict that the most massive subhaloes of the Milky way are too dense to host any of its bright satellites, with luminosity higher than $10^{5}$ the luminosity of the Sun [20].

All three problems have their root in the fact that the cold DM particles, which the $\Lambda$ CDM simulations rely upon, have too short free streaming length during the epochs of galaxy formation, and therefore they form too clumped and too many structures compared to those observed.

Understanding the shape and depth of the gravitational potential in dSph may have an important bearing in the understanding of these fundamental questions. In this respect we mention a new 


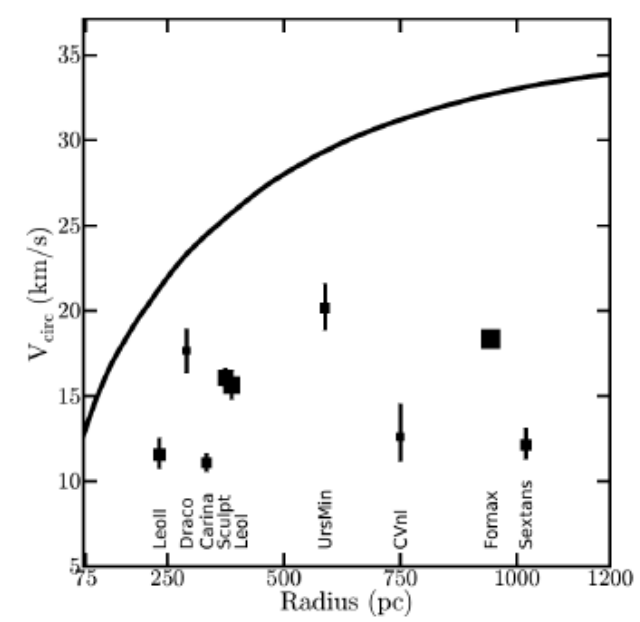

Figure 1: The "too-big-to-fail problem": the continuous line denotes the rotation curve of typical largest subhalo of the Milky Way Galaxy, as simulated within the collisionless $\Lambda$ CDM model. The data points pertain to observed circular velocities of the largest subhalos of the Milky Way at their half light radii. The discrepancy is obvious. Picture taken from Ref. [20].

method for estimating the $\mathrm{dSph}$ gravitational potential [21], based on exploiting the higher order analogues of the projected Virial Theorem $2 K_{z}+W_{s}=0$, which provide global constraints on the moments of the velocity distribution $\left(\left\langle v_{z}^{n}\right\rangle\right)$ by effectively integrating the spherical Jeans equations over all radii:

$$
\begin{aligned}
\text { second order : } \int_{0}^{\infty} \Sigma(R)\left\langle v_{z}^{2}\right\rangle R d R & =\frac{2}{3} \int_{0}^{\infty} v \frac{d \Phi}{d r} r^{3} d r \\
\text { fourth order : } \int_{0}^{\infty} \Sigma(R)\left\langle v_{z}^{4}\right\rangle R d R & =\frac{2}{5} \int_{0}^{\infty} v(5-2 \beta)\left\langle\mathrm{v}_{r}^{2}\right\rangle \frac{d \Phi}{d r} r^{3} d r, \\
\int_{0}^{\infty} \Sigma(R)\left\langle v_{z}^{2}\right\rangle R^{3} d R & =\frac{4}{35} \int_{0}^{\infty} v(7-6 \beta)\left\langle\mathrm{v}_{r}^{2}\right\rangle \frac{d \Phi}{d r} r^{5} d r,
\end{aligned}
$$

where $r$ denotes the physical (three-dimensional (3D)) radius of a star from the centre of its galaxy, $v(r)$ is the 3D number density of stars, $R$ and $\Sigma(R)=\int_{-\infty}^{\infty} v(r) d z$ are projections of $r$ and $v$, respectively, onto the plane perpendicular to the line of sight (LOS); $\Phi$ is the gravitational potential and $\beta=\beta(r)=1-\frac{\sigma_{t}^{2}(r)}{\sigma_{r}^{2}(r)}$ is the stellar velocity anisotropy parameter, with $\sigma_{r}^{2}\left(\sigma_{t}^{2}=2 \sigma_{\theta}^{2}=2 \sigma_{\phi}^{2}\right)$ is the variance of the radial (tangential) velocity distributions. The quantities (2.1) carry more or less the same information as the corresponding Jeans equations [21]. Based on such novel approaches, and defining appropriate Virial shape estimators from (2.1), the authors of Ref. [21] performed an illustrative analysis on the DM profile of the galaxy Sculptor, NGC 253, in the Sculptor group, using phenomenological expressions for $v(r)$ and the density profile $\rho(r)$, which is taken to be close to the NFW. This galaxy is a good study case, given that it is one of the brightest galaxies in the vicinity of the Milky Way, and is a starburst, that is it undergoes intense star formation, which according to several astronomers it is believed to have been caused by the collision with a dwarf galaxy a few hundred million years ago, disturbing its disc and started the currently observed starburst. There is also a belief that there is a supermassive black hole at its centre which is slightly heavier that that of 
the centre of the Milky Way, Sagittarius A* (Sgr A*). The novel analysis of Ref. [21], where more general functions of $\beta(r)$ have been used, has demonstrated that one can fit the velocity dispersion of Sculptor using NFW profiles, leading to the conclusion that, contrary to the results of other analyses based on traditional Jeans equations, there is no extended core in the Sculptor in agreement with $\Lambda \mathrm{CDM}$ simulations. Although from such a single study case one cannot make generic conclusions, nevertheless one would be tempted to conjecture that the dwarf spheroidals do not have cores, in which case there should be no discrepancy with $\Lambda$ CDM simulations [21]. However, this point of view is not shared by the majority of the astrophysicists.

Adopting the standard point of view, several possible solutions to the core-cusp problem have been proposed. Many recent studies have shown that including baryonic feedback (particularly feedback from supernovae and active galactic nuclei) can "flatten out" the core of a galaxy's dark matter profile, since feedback-driven gas outflows produce a time-varying gravitational potential that transfers energy to the orbits of the collisionless dark matter particles [22]. Other works have shown that the core-cusp problem can be solved outside of the most widely accepted Cold Dark Matter (CDM) paradigm: simulations with warm or self-interacting dark matter (SIDM) also produce dark matter cores in low-mass galaxies [23].

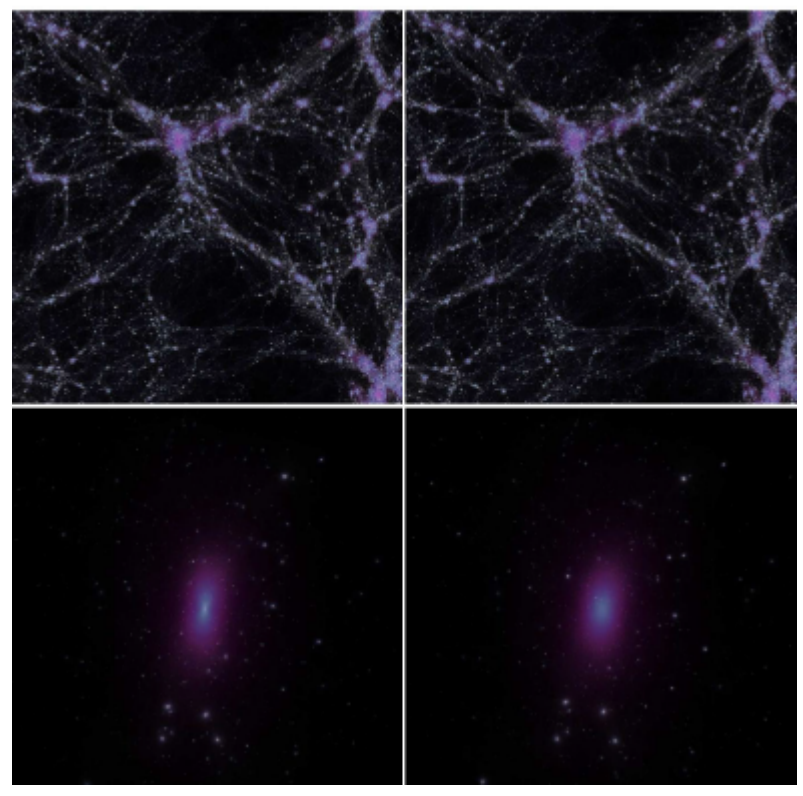

Figure 2: Comparison of coillisionless $\Lambda C D M$ model simulations (left panels) with Sel-interacting Dark Matter (SIDM) simulations with cross section $\sigma=1 \mathrm{~cm}^{2} / \mathrm{g}$ (right panels). The upper panels pertain to large scale structure, where the two models agree, while the lower panels refer to individual galaxies, where one observes that in SIDM models, galaxies appear more cored and spherical. Pictures taken from Ref. [27].

The missing satellite problem has two potential solutions [24]. One is that the smaller halos do exist but only a few of them end up becoming visible because they have not been able to attract enough baryonic matter to create a visible dwarf galaxy. In support of this, Keck observations in 2007 of eight newly discovered ultra-faint Milky Way dwarf satellites showed that six were almost exclusively composed of DM, around 99.9\% (with a mass-to-light ratio of about 1000) [25]. Such ultra-faint dwarfs substantially alleviate the discrepancy between the predicted and observed 
numbers of satellites around the Milky Way, but there are still discrepancies by a factor of about four too few dwarf galaxies over a significant range of masses. In Ref. [25], the authors argued that, if galaxy formation in low-mass dark matter halos is strongly suppressed after re-ionization, then the simulated circular velocity function of CDM subhalos can be brought into approximate agreement with the observed circular velocity function of Milky Way satellite galaxies. Other solutions may be that dwarf galaxies tend to be merged into or tidally stripped apart by larger galaxies due to complex interactions. This tidal stripping has been part of the problem in identifying dwarf galaxies in the first place, which is an extremely difficult task since these objects have low surface brightness and are highly diffused, so much that they are virtually unnoticeable.

Finally, the Too-Big-to-Fail Problem may also be tackled by the combined inclusion of self interactions in DM, which tend to make the individual galaxies more cored and spherical, along with baryonic feedback [13].

At this point we stress that self interactions have been argued to play an important rôle in galactic structure already in Ref. [26]. The original idea of self-interacting DM (SIDM) was implemented for CDM particles with rest masses above $1 \mathrm{MeV} / c^{2}$ (up to $10 \mathrm{GeV} / c^{2}$ ), consistent with the nature of the effective interactions and the mean free paths considered in that work. This way of thinking regarding self-interactions was applied uniquely on DM halo scales with typical densities of $10^{-2} M_{\odot} / \mathrm{pc}^{3}$, suggesting that normalized total cross-sections of order $\sigma / m \sim 0.1-100 \mathrm{~cm}^{2} / \mathrm{g}$, would imply observational effects in the inner regions of the DM halos. It was also shown that a SIDM regime with these values of $\sigma / m$ would generate shallower inner DM profiles, with a necessary reduction in the amount of sub-structures, thereby alleviating (or even solving) the core-cusp and the missing satellite problems of collisionless $\Lambda \mathrm{CDM}$, as mentioned above. However, contemporaneously, some tension with upper limits in the DM cross sections as obtained from lensing studies on galaxy cluster scales emerged. In a subsequent work [27], motivated by updated analysis of the Bullet Cluster [28], new cosmological simulations within CDM were performed, with the aim of further scrutinizing the effects of SIDM on inner halo cores of galaxies and galaxy clusters.

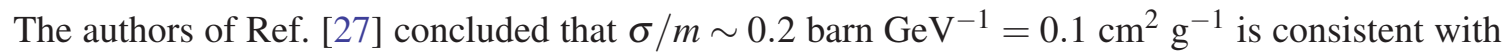
all the observational constraints. In general, SIDM would make no difference from $\Lambda$ CDM at large scales, but individual galaxies would appear more cored and spherical, with higher velocity dispersion ( $c f$. Fig. 2). As emphasised in ref. [13], observations from clusters, such as the aforementioned refined analyses in the bullet cluster [28], constitute important tools to probe self-interacting DM models.

Self interactions among DM particles could tackle the core-cusp and too big to fail problems by reducing the central density. Stringent constraints can be imposed on the pertinent interaction cross sections of SIDM by studying merging (colliding) galaxies [13]. In this latter respect we mention the works of Ref. [29], according to which self interactions can lead to both deceleration and evaporation of a DM halo when the latter moves through a background of DM particles. This results in a shift of the halo's centroid relative to the collisionless stars and galaxies. The substructure evaporation phenomenon is rare, and may be attributed to a short range dark force, e.g. due to a massive non-Abelian vector dark boson exchange graph in $\chi \chi \rightarrow \chi \chi \mathrm{DM}$ scattering. This leads to isotropic scattering cross sections $\sigma$. Au contraire, the exchange of long-range dark photons in the above self-interaction, is frequent, leading to low-momentum transfer and directional scattering, in the sense that the cross section is no longer isotropic but depends on the scattering angle $\theta, \sigma(\theta)$. 
This leads to substructure deceleration. By concentrating on such phenomena, one may impose limited constraints on SIDM cross sections by the (conventional) study of several merging clusters of galaxies, without taking into account DM drag during the collision.

However, by defining new observables, taking into account the DM drag [13], yields more stringent constraints on the self-interaction DM cross section. In particular, the study of 72 mergers using this new technique imposed a more stringent constraint on the SIDM cross section per unit DM mass $\sigma / m \leq 0.47 \mathrm{~cm}^{2} / \mathrm{g}$, which together with the lower bound $\sigma / m \geq 0.1 \mathrm{~cm}^{2} / \mathrm{g}$ imposed by the cosmology on galaxy scales, defines a new range for $\sigma_{\text {SIDM }} / m[13]$

$$
0.1 \leq \frac{\sigma_{\mathrm{SIDM}} / m}{\mathrm{~cm}^{2} \mathrm{~g}^{-1}} \leq 0.47
$$

to be considered in galactic studies. This leads to a possible resolution of the three "small scale cosmology problems" of DM.

A challenge, and some tension with the upper bound of (2.2) still remains [30], as a result of observations in the Abell Cluster 3827. The observed separation between the dark matter halo and the stars of a galaxy moving through a region of large dark matter density (i.e. the core of Abell 3827 in this case), which is a characteristic feature of SIDM, suggests that this cluster may provide the first evidence of a SIDM [13]. However in Ref. [30], the authors estimated the DM selfinteraction cross section needed to reproduce the observed effects, and argued that the sensitivity of Abell 3827 has been significantly overestimated in a previous study [31]. In fact, their basic point was that the model used in Ref. [31] to interpret the observations in terms of DM self interactions failed to take into account the non independent development of the stars and the DM subhalo due to the initial gravitational bound of the former to the latter. Indeed, to achieve a star-subhalo separation, as observed, one would need external forces (such as self interactions) comparable in strength to the gravitational attraction within the system. Another feature of the model used in Ref. [31] which the authors of Ref. [30] criticised, was the assumption that the effective DM drag force was constant throughout the evolution of the system. Such a feature might have been expected if the subhalo were on a circular orbit along the trajectory of the Abell 3827 cluster, wich however is disfavoured by observations. Moreover, the constant-drag-force assumption also contradicts the fact that a typical rate of DM self interactions depends on the velocity of the subhalo relative to the cluster, as well as the DM density of the cluster. Both vary along the subhalo trajectory, and in fact the rate of DM self interactions is negligibly small, as long as the subhalo is far away from the core of the cluster. The corrected estimate for the SIDM cross section per DM mass in the analysis of Ref. [30] is $\sigma_{\mathrm{SIDM}} / m \simeq 3 \mathrm{~cm}^{2} \mathrm{~g}^{-1}$, when self interactions result in a drag force, and $\sigma_{\mathrm{SIDM}} / m \simeq 1.5 \mathrm{~cm}^{2} \mathrm{~g}^{-1}$ in the case of contact interactions, in tension with the upper bounds (2.2).

The above example of Abell 3827 shows how active in research and yet inconclusive, due to both theoretical and observational challenges, is the field of self interactions of DM from the generic astrophysical view point. Notice however that no attempt is made in the above discussions to analyze microscopic interactions that may lead to phenomenologically acceptable SIDM cross sections. A concrete example of SIDM $[14,15]$ is based on a class of self-interacting models of massive right-handed neutrinos that exist in minimal extensions of the standard model. It is important to stress that, in contrast to standard N-body simulations, the semi-analytical approach proposed in Refs. [14, 15] includes in addition to gravity, other important physical ingredients, such 
as quantum (fermionic) physics and thermodynamics. Interestingly, such a scenario, in which the DM fermion (right-handed neutrino) has a mass of a order a few tens of $\mathrm{keV}$, implies a universal and novel DM density profile (compact core - dilute halo), with important implications for the very central and halo regions of galaxies ${ }^{2}$. We shall discuss this model and its rôle in resolving the aforementioned three-problems of small scale cosmology in the next section.

\section{Self-interacting right-handed neutrino \& DM distribution in galaxies}

The right-handed neutrino model for SIDM of Ref. [14] is based on minimal (non supersymmetric) extensions of the Standard Model with sterile neutrinos. In such models, the DM may be provided by the lightest $N_{R 1}$ of three right-handed neutrino species that, e.g. appear in the $v$ MSM model [33], but this identification is not binding. However, unlike $v$ MSM, we allow our right-handed neutrinos to be self-interacting. We introduce phenomenologically neutrino self-interactions through a massive-vector-meson $V_{\mu}$ mediator. For concreteness we assume the fermions to be of Majorana type (nevertheless, the formalism is readily extendable to Dirac fermions). This is the common feature our model shares with the $v$ MSM. As we shall argue in this work, there is an intriguing similarity in the allowed range (in the few tens of $\mathrm{keV}$ ) of the sterile neutrino DM mass between the two models, despite the fact that these bounds have been obtained by quite different reasons.

The Lagrangian of the right-handed neutrino sector, including gravity, reads (in units $\hbar=c=1$, which we use throughout here) [14]

$$
\mathscr{L}=\mathscr{L}_{G R}+\mathscr{L}_{N_{R 1}}+\mathscr{L}_{V}+\mathscr{L}_{I}
$$

where

$$
\begin{aligned}
\mathscr{L}_{G R} & =-\frac{R}{16 \pi G}, \quad \mathscr{L}_{N_{R 1}}=i \bar{N}_{R 1} \gamma^{\mu} \nabla_{\mu} N_{R 1}-\frac{1}{2} m \bar{N}_{R 1} N_{R 1}, \\
\mathscr{L}_{V} & =-\frac{1}{4} V_{\mu \nu} V^{\mu \nu}+\frac{1}{2} m_{V}^{2} V_{\mu} V^{\mu}, \quad \mathscr{L}_{I}=-g_{V} V_{\mu} J_{V}^{\mu}=-g_{V} V_{\mu} \bar{N}_{R 1} \gamma^{\mu} N_{R 1},
\end{aligned}
$$

where $m$ is the mass of the sterile neutrino, with $R$ the Ricci scalar for the static spherically symmetric metric background $g_{\mu v}=\operatorname{diag}\left(e^{v},-e^{\lambda},-r^{2},-r^{2} \sin ^{2} \varphi\right)$, where $e^{v}$ and $e^{\lambda}$ depend only on the radial coordinate; $r$ and $\varphi$ denotes the polar angle. The quantity $\nabla_{\mu}=\partial_{\mu}-\frac{i}{8} \omega_{\mu}^{a b}\left[\gamma_{a}, \gamma_{b}\right]$ is the gravitational covariant derivative acting on a Majorana spinor, with $\omega_{\mu}^{a b}$ the spin connection. The right-handed sterile neutrinos $N_{R 1}$ satisfy the Majorana four-spinor condition, $\Psi^{c}=\Psi$, together with $\bar{\Psi}=\Psi^{T} C$, where the conjugate spinor field $\Psi^{c}=C \bar{\Psi}^{T}$ and $C$ is the unitary $\left(C^{\dagger}=C^{-1}\right)$ charge conjugation operator, flipping the fermion chirality, i.e. $\left(\Psi_{L}\right)^{c}=\left(\Psi^{c}\right)_{R}$ is right-handed (R), whilst $\left(\Psi_{R}\right)^{c}=\left(\Psi^{c}\right)_{L}$ is left-handed (L). The definition of chirality (handedness) is the standard one, $\Psi_{L(R)}=\frac{1}{2}\left(1 \mp \gamma^{5}\right) \Psi$, with the $+(-)$ sign denoting Right-(Left)handed spinors, and $\gamma_{5}=i \gamma^{0} \gamma^{1} \gamma^{2} \gamma^{3}$, with $\gamma^{\mu}$ the $4 \times 4$ Dirac matrices. The massive-vector-mesons $V_{\mu}$ should not be viewed as gauge bosons if the fermions are Majorana. As is well known, the Lorentz gauge condition $\partial^{\mu} V_{\mu}=0$,

\footnotetext{
${ }^{2}$ Similar core - halo DM density behaviour have also been obtained within modern quantum-wave dark matter 3D N-body simulations [32]
} 
which we assume here, emerges in that case as a consequence of their equations of motion. Latin indices denote pertain to flat tangent space and hence they are raised and lowered with the Minkowski $\eta_{a b}$ metric. The microscopic origin of the vector meson mass $m_{V}$ is not discussed here. It may well come from an appropriate Higgs mechanism in the dark sector. For simplicity we assume minimalcoupling of the vector field with the sterile neutrino current $J_{V}^{\mu}$ in the interaction term $\mathscr{L}_{I}$ (3.2). This current is conserved if decays of sterile neutrinos are ignored. Such a coupling may also arise from linearisation of a Thirring-type four fermion vector current interaction $J_{V}^{\mu} J_{V \mu}$ by means of an auxiliary vector field $A_{\mu}$ (which acquires dynamics upon implementing quantum corrections). Such a four-fermion-contact-interaction model is also the effective low-energy approximation of the detailed model (3.2) for fermion energies much lower than the scale $m_{V}$.

In general one may add to (3.1) a Yukawa term, coupling the (three, in general) right-handed neutrinos to the active neutrino sector (see, e.g., the case of $v$ MSM in Ref. [33])

$$
\mathscr{L}_{\text {Yuk }}=F_{\alpha I} \bar{\ell}_{\alpha} N_{R I} \phi^{c}+\text { h.c. }, \quad I=1,2,3
$$

where $\ell_{\alpha}$ are the lepton doublets of the SM, $\alpha=e, \mu, \tau, F_{\alpha I}$ are appropriate Yukawa couplings, and $\phi^{c}$ is the SM conjugate Higgs field, i.e. $\phi^{c}=i \tau_{2} \phi^{\star}$, with $\tau_{2}$ the $2 \times 2$ Pauli matrix. Upon considering such a coupling, one obtains the stringent X-ray and BBN constraints of the mixing angle and mass of $N_{R 1}$, given that (3.3) implies decays of the heavy neutrinos $N_{I} \rightarrow v H$, where $H$ denotes the Higgs excitation field, defined via: $\phi=\langle\phi\rangle+H$. In such a case $J_{V}^{\mu}$ is not conserved in time. However, in the context of $v \mathrm{MSM}$, the lightest of the heavy neutrinos decay time is longer than the age of the universe, hence the latter can be considered as stable for all practical purposes, thus playing the rôle of DM. For our purposes, as already mentioned, we concentrate here on this lightest neutrino and ignore such a mixing with the SM sector, setting $F_{\alpha 1}=0$, in which case the lightest neutrino is absolutely stable ${ }^{3}$. The important feature for us are the self-interactions of the right-handed neutrino, which will be used for ensuring phenomenologically correct values for the radius and mass of the galactic core. The analysis in Ref. [14] has been performed within a relativistic mean field (RMF) approximation, according to which the system can be considered as corresponding to a static uniform matter distribution in its ground state. Information about the strength of the self-interaction coupling of the effective interactions of the fermions ('inos') and the mass of the vector-meson mediator is encoded in the quantity

$$
C_{V} \equiv g_{V}^{2} / m_{V}^{2}
$$

which determines the order of magnitude of the corresponding cross section, as we shall explain below. As we have discussed above, this is important for the small-scale DM distributions. In Ref. [14] it was assumed that the self-interactions among the DM neutrinos occur only in the

\footnotetext{
${ }^{3}$ The inclusion of such interactions do not affect our conclusions, as justified in detail in Ref. [14]. This is due to the very weak nature of the Yukawa coupling $F_{\alpha I}$, necessitated by the seesaw mechanism. However, an interesting motivation to include coupling of the right-handed neutrinos with the SM sector (active) neutrinos $v$, is to be able to obtain a possible indirect detection method through the decaying channel $N_{R 1} \rightarrow v+\gamma$, with a potential enhancement due to their self-interacting nature. In this respect we cannot resist in pointing out the recent observations by the Fermi satellite, providing evidence of a clear emission in the energy range 10-25 keV from the central region of the Galaxy [34]. The latter could find a plausible explanation by means of a DM particle species with a mass of order $50 \mathrm{keV} / \mathrm{c}^{2}$, similar to the one obtained in Ref. [14] and discussed here.
} 
quantum regime and thus within the core, where the thermal de-Broglie wavelength $\lambda_{B}=\frac{h}{\sqrt{2 \pi m k_{B} T}}$ is larger than the inter-particle mean distance $l$ at temperature $T, \lambda_{B} / l>1$. That is, we consider the ansatz:

$$
C_{V}(r)=\left\{\begin{array}{llll}
C_{0} & \text { at } \quad r<r_{m} & \text { when } & \lambda_{B} / l>1 \\
0 & \text { at } \quad r \geqslant r_{m} & \text { when } & \lambda_{B} / l<1
\end{array}\right.
$$

where $C_{0}$ is a positive constant and $r_{m}=r_{c}+\delta r$, with $\delta r \ll r_{c}$, is the core-halo matching point, with $r_{c}$ the core radius and $\delta r$ the thickness of the core-halo intermediate layer.[14] The region $r \geqslant r_{m}$, where the DM distribution is in a much more dilute state (i.e. $\lambda_{B} / l \ll 1$ ), marks the transition from the quantum degenerate state to the Boltzmann one.
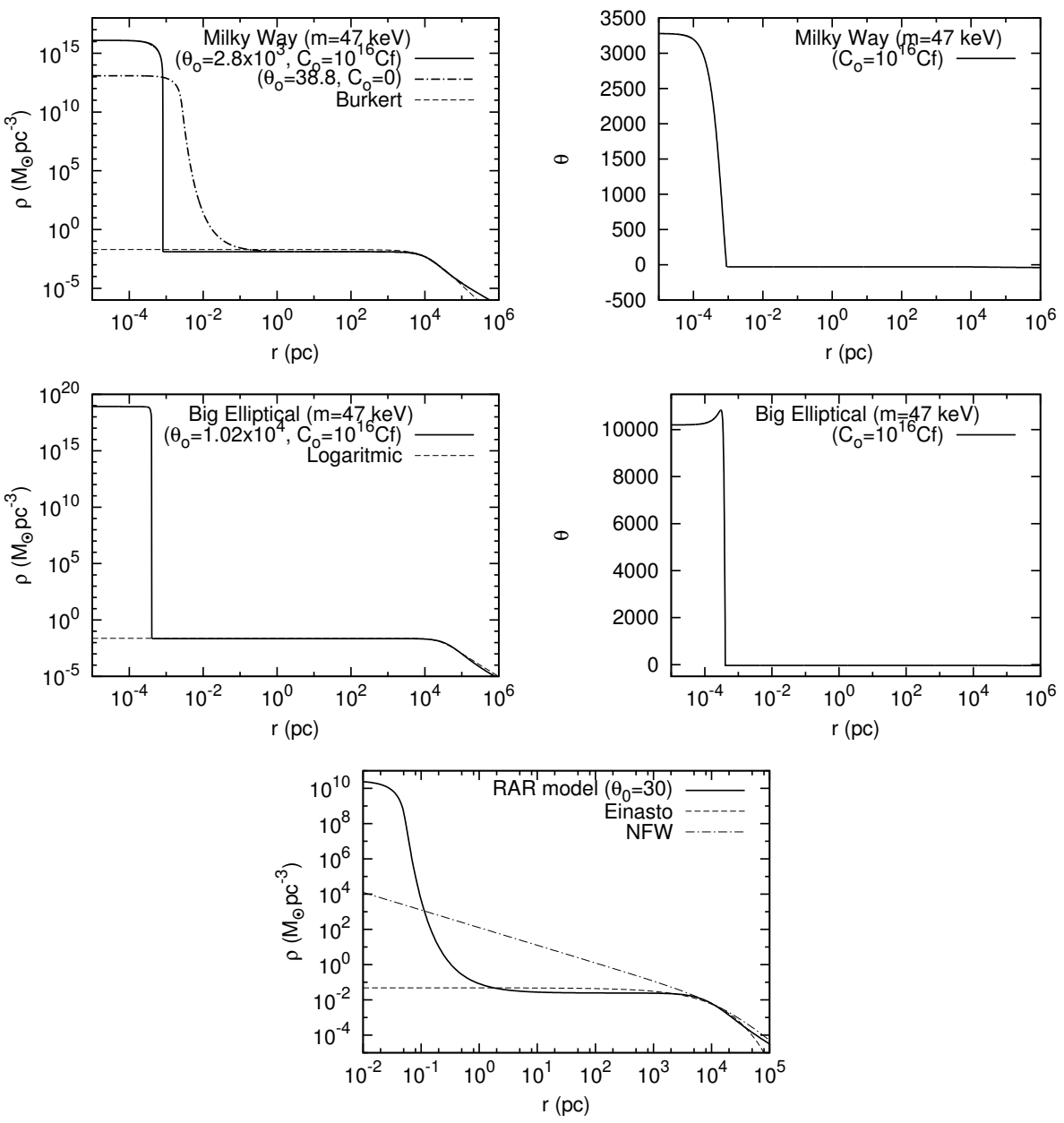

Figure 3: First two upper panels: Mass density $\rho$ and degeneracy $\theta$ profiles (vs distance $r$ from the centre of the galaxy) for $m=47 \mathrm{keV} / \mathrm{c}^{2}$ in the interaction regime $\bar{C}_{0}=10^{16}$, where core and halo Milky Way observational constraints are fulfilled, compared with the non-interacting case $\left(\bar{C}_{0}=0\right)$ for the same ino mass. Both the interacting and non-interacting cases use the Ruffini-Argüelles-Rueda (RAR) DM density profile,[15] which is in good agreement with Burkert profile. Middle two panels: The same as in previous case but for the case of a large elliptical galaxy. Lower panel: The RAR profile (in the non-interacting case $\left.C_{V}=0\right)$ and its comparison with the NFW and a cored Einasto profile for a typical spiral galaxy. The picture at the bottom panel was taken from Ref. [15], while the rest from Ref. [14]. 
There are three free parameters in the approach, evaluated at the galactic centre: the DM temperature per unit mass $\left(\beta_{0}=k_{B} T_{0} / m\right)$, the degeneracy parameter at the centre (depending on the chemical potential $\left.\mu_{0}\right) \theta_{0}=\mu_{0} /\left(k_{B} T_{0}\right)$, and the self-interaction constant $C_{0}$ (coupling). The analysis of ref. [14] can constrain the degeneracy parameters $\beta \equiv k_{B} T / m=\beta_{0} e^{\left(v_{0}-v(r)\right) / 2}$ and $\theta \equiv \mu /\left(k_{B} T\right)$ at the core $\left(\beta_{0}, \theta_{0}\right)$, together with the the sterile neutrino mass $m$ and coupling $C_{V}$. The relevant constraints are given in Table 1 . The corresponding galaxy density profiles and degeneracy parameters are depicted in figure 3, where for comparison (in the bottom panel) we also plot the corresponding results for the non interacting case $\left(C_{V}=0\right)$ discussed in Ref. [15]. The calculations were done for the maximum allowed range of the interaction constant $\bar{C}_{0}$, and the corresponding central degeneracy $\theta_{0}$, temperature $\beta_{0}$ and ino mass $m$.

\begin{tabular}{c|c|c|c|c|c|c}
\multicolumn{7}{c}{ Milky Way $\left(M_{c}=4.4 \times 10^{6} M_{\odot}\right)$} \\
\hline$m(\mathrm{keV})$ & $\bar{C}_{0}$ & $\theta_{0}$ & $\beta_{0}$ & $r_{c}(\mathrm{pc})$ & $\delta r(\mathrm{pc})$ & $\theta\left(r_{m}\right)$ \\
\hline 47 & 2 & $3.70 \times 10^{3}$ & $1.065 \times 10^{-7}$ & $6.2 \times 10^{-4}$ & $2.1 \times 10^{-4}$ & -29.3 \\
& $10^{14}$ & $3.63 \times 10^{3}$ & $1.065 \times 10^{-7}$ & $6.2 \times 10^{-4}$ & $2.2 \times 10^{-4}$ & -29.3 \\
& $10^{16}$ & $2.8 \times 10^{3}$ & $1.065 \times 10^{-7}$ & $6.3 \times 10^{-4}$ & $2.4 \times 10^{-4}$ & -29.3 \\
\hline 350 & 1 & $2.40 \times 10^{6(\dagger)}$ & $1.431 \times 10^{-7}$ & $1.3 \times 10^{-6}$ & $6.7 \times 10^{-7}$ & -37.3 \\
& $10^{14}$ & $1.27 \times 10^{5}$ & $1.104 \times 10^{-7}$ & $5.9 \times 10^{-6}$ & $9.4 \times 10^{-7}$ & -37.3 \\
& $4.5 \times 10^{18}$ & $1.7 \times 10^{1}$ & $1.065 \times 10^{-7}$ & $5.9 \times 10^{-4}$ & $2.0 \times 10^{-4}$ & -37.3 \\
\hline \multicolumn{7}{c}{ Elliptical $\left(M_{c}^{c r}=2.3 \times 10^{8} M_{\odot}\right)$} \\
\hline 47 & 2 & $1.76 \times 10^{5(\dagger)}$ & $1.7 \times 10^{-6}$ & $7.9 \times 10^{-5}$ & $3.9 \times 10^{-5}$ & -31.8 \\
& $10^{14}$ & $5.8 \times 10^{4}$ & $1.4 \times 10^{-6}$ & $1.4 \times 10^{-4}$ & $4.8 \times 10^{-5}$ & -31.8 \\
& $10^{16}$ & $1.5 \times 10^{4}$ & $1.3 \times 10^{-6}$ & $3.0 \times 10^{-4}$ & $7.0 \times 10^{-5}$ & -31.8 \\
\hline \multicolumn{7}{c}{ Large Elliptical $\left(M_{c}=1.8 \times 10^{9} M_{\odot}\right)$} \\
\hline 47 & $10^{16}$ & $1.02 \times 10^{4}$ & $3.0 \times 10^{-6}$ & $3.8 \times 10^{-4}$ & $1.8 \times 10^{-5}$ & -32.8 \\
\hline
\end{tabular}

Table 1: Set of right-handed SIDM model parameters for three different galaxy types analyzed in Ref. [14] that satisfy all the appropriate core and halo conditions.

From the results presented in Table 1 one can see that for DM mass $m<47 \mathrm{keV} / c^{2}$ or $m>$ $350 \mathrm{keV} / c^{2}$ there is no pair of parameters $\left(\bar{C}_{0}, \theta_{0}\right)$ that can fit the Milky Way observables. On the other hand, $m=47 \mathrm{keV} / c^{2}$ is the lower bound for the particle mass that satisfies the observed core constraints (within the observational errors), while $m=350 \mathrm{keV} / c^{2}$ is the uppermost bound set by reaching the critical core mass for gravitational collapse, $M_{c}^{c r} \propto M_{\mathrm{Pl}}^{3} / m^{2} \approx 4.4 \times 10^{6} M_{\odot}$ (see Ref. [35]). From figure 3, one can also see that the inclusion of sufficiently strong interactions in the dark sector among the sterile neutrinos can lead to significantly more compact cores and higher central degeneracies than the free case. The total $N_{R 1}-N_{R 1}$ scattering cross-section in the quantum core of the Galaxy has been calculated in Ref. [14] in a perturbative regime $g_{V}<1$ for the dimensionless interaction coupling:

$$
\sigma_{\text {core }}^{\text {tot }} \approx \frac{\left(g_{V} / m_{V}\right)^{4}}{4^{3} \pi} 29 m^{2} \quad\left(p^{2} / m^{2} \ll 1\right) .
$$

To put things in perspective, one can normalize the interaction field strength in terms of the visible sector (SM) weak interaction dimensionfull coupling, the Fermi "constant" $G_{F}$, by defining and 
estimating the quantity $\bar{C}_{V}=\left(\frac{g_{V}}{m_{V}}\right)^{2} G_{F}^{-1}$, as done in Ref. [14]. Thus, if, e.g. one constrains the total cross-section to the N-body simulation value $\sigma^{t o t} / m=0.1 \mathrm{~cm}^{2} / \mathrm{g}$ (or in general to lie in the region (2.2)), the coupling constant $\bar{C}_{V}$ would be constrained to the value

$$
\bar{C}_{V} \in\left(2.6 \times 10^{8}, 7 \times 10^{8}\right),
$$

for right-handed neutrino masses in the range $m \in(47,350) \mathrm{keV}$. It worths noticing that for $C_{V} \sim$ $10^{8} G_{F}$, the mass of the massive-vector meson would be constrained to values $m_{V} \lesssim 3 \times 10^{4} \mathrm{keV}$, in order to satisfy $g_{V} \lesssim 1$ as requested by the self-consistency of the perturbation scheme we have applied to compute the cross-section. A conservative lower bound of $C_{V}$ has been obtained in Ref. [14] by requiring that the cross section $\sigma$ be sufficiently large so that a scattering probability among the inos occurs at least once during the age of the galaxy:

$$
\sigma / m \gtrsim 10^{-18} \mathrm{~cm}^{2} / \mathrm{g}
$$

implying from (3.6) that $C_{V} \gtrsim 2 G_{F}$.

\section{Conclusions and outlook}

In this work we have emphasized the rôle of self interactions in the dark matter (SIDM) sector of the universe in bridging the gap between observations and the numerical simulations based on the $\Lambda \mathrm{CDM}$ paradigm. We have discussed briefly how interacting dark matter models may tackle the three basic challenges for N-body simulations based on $\Lambda \mathrm{CDM}$ cosmology, associated with the DM distribution in galaxies ("small-scale Cosmology crisis"). We have also seen how the situation at present is far from being conclusive, given that there are always observed cases, like the aforementioned Abell 3827 cluster, which challenge even the most successful of models. Dark matter may, like matter, consist of more than one dominant species, which co-exist harmonically at various scales, in such a way that, for instance CDM may provide a good explanation for large scale structure, but other species, such as right-handed neutrinos, play also an important rôle for explaining the observed details in core-halo structures of a galaxy. One should also bear in mind that more mundane astrophysical explanations may be in operation together with DM self interactions, in order to account for a complete set of observations.

As stressed in Ref. [14], self-interactions among WDM right-handed Neutrinos, in minimal extensions of the Standard Model, may provide solution to all three problems of the small-scale cosmology, and model more accurately the galactic structure. The inclusion of baryonic matter in this picture is not expected to change the basic conclusion that the introduction of WDM fermion self interactions affects the core/halo structure and in particular induces higher central degeneracies and higher compactness of the inner quantum core of galaxies. Moreover, the model provides a natural resolution to the core-cusp problem of DM at small scales, because the density profiles based on fermionic phase-space distributions develop always an extended plateau on halo scales, in a way that resemble Burkert or cored Einasto profiles (cf. Fig. 3). Another important feature is the fact that the right-handed neutrino DM mass is 'colder' by a few $\mathrm{keV}$ as compared to most of the WDM models available in the literature, which implies that the model does not suffer from 
standard WDM problems (see for instance the discussion on exclusion of WDM mentioned in the introduction of the review).

A final observation regards the range of the self-interacting DM neutrino masses, $m \geq 47$ $\mathrm{keV} / \mathrm{c}^{2}$. As stressed in Ref. [14], by identifying the right-handed DM Majorana neutrinos of the model with the (lightest) right-handed neutrino of the $v$ MSM model [33], our SIDM should have a very weak mixing angle with the SM lepton sector, and its mass should be less than $50 \mathrm{keV} / \mathrm{c}^{2}$, otherwise the model would not be consistent with current cosmology [33]. The above considerations, then, leave a very narrow range of the mass $47 \leq m \leq 50 \mathrm{keV} / c^{2}$ for the self-interacting right-handed neutrino to play both rôles, that of a WDM candidate and that of a provider of a corehalo galactic structure in agreement with observations. It becomes therefore interesting to look for independent tests of this model, for instance, in neutrino oscillations or other relevant particle physics experiments like SHIP [36].

\section{References}

[1] M. S. Longair, Galaxy formation (Berlin: Springer, 2008), ,ISBN 978-3-540-73477-2

[2] G. Bertone, Particle Dark Matter: Observations, Models and Searches (Cambridge University Press. 2010), ISBN 978-0-521-76368-4.

[3] P. A. R. Ade et al. [Planck Collaboration], Planck 2015 results. XIII. Cosmological parameters, arXiv:1502.01589 [astro-ph.CO].

[4] D. N. Spergel et al. [WMAP Collaboration], First year Wilkinson Microwave Anisotropy Probe (WMAP) observations: Determination of cosmological parameters, Astrophys. J. Suppl. 148, 175 (2003) doi:10.1086/377226 [astro-ph/0302209]; G. Hinshaw et al. [WMAP Collaboration], Nine-Year Wilkinson Microwave Anisotropy Probe (WMAP) Observations: Cosmological Parameter Results, Astrophys. J. Suppl. 208 (2013) 19 doi:10.1088/0067-0049/208/2/19 [arXiv:1212.5226 [astro-ph.CO]].

[5] A. Loeb, and M. Zaldarriaga, Phys. Rev. D 71, 103520 (2005) doi:10.1103/PhysRevD.71.103520 [astro-ph/0504112].

[6] P. Bode, J. P. Ostriker, and N. Turok, Astrophys. J. 556, 93 (2001) doi:10.1086/321541 [astro-ph/0010389].

[7] N. Yoshida, A. Sokasian, L. Hernquist and V. Springel, Astrophys. J. 591, L1 (2003) doi:10.1086/376963 [astro-ph/0303622].

[8] M. Viel, G. D. Becker, J. S. Bolton, and M. G. Haehnelt, Phys. Rev. D 88, 043502 (2013) doi:10.1103/PhysRevD.88.043502 [astro-ph/1306.2314]

[9] U. Seljak, A. Slosar and P. McDonald, JCAP 0610, 014 (2006) doi:10.1088/1475-7516/2006/10/014 [astro-ph/0604335].

[10] see, for instance: A. B. Lahanas, N. E. Mavromatos and D. V. Nanopoulos, Int. J. Mod. Phys. D 12, 1529 (2003) doi:10.1142/S0218271803004286 [hep-ph/0308251].

[11] E. W. Kolb and M. S. Turner, "The Early Universe" Front. Phys. 69, 1 (1990).

[12] B. S. Acharya, G. Kane, S. Watson and P. Kumar, Phys. Rev. D 80, 083529 (2009) doi:10.1103/PhysRevD.80.083529 [arXiv:0908.2430 [astro-ph.CO]]. 
[13] D. Harvey, R. Massey, T. Kitching, A. Taylor, E. Tittley Science 347, 6229, 1462 (2015) and references therein.

[14] C. R. Argüelles, N. E. Mavromatos, J. A. Rueda and R. Ruffini, JCAP 04 (2016) 038 http://dx.doi.org/10.1088/1475-7516/2016/04/038 arXiv:1502.00136 [astro-ph.GA].

[15] R. Ruffini, C. R. Argüelles and J. A. Rueda, Mon. Not. Roy. Astron. Soc. 451, no. 1, 622 (2015) doi:10.1093/mnras/stv1016 [arXiv:1409.7365 [astro-ph.GA]]. See also: I. Siutsou, C. R. Argüelles and R. Ruffini, Astron. Rep. 59, no. 7, 656 (2015) doi:10.1134/S1063772915070124 [arXiv:1402.0695 [astro-ph.GA]]; C. R. Argüelles, R. Ruffini, I. Siutsou and B. Fraga, J. Korean Phys. Soc. 65, no. 6, 801 (2014) doi:10.3938/jkps.65.801 [arXiv:1402.0700 [astro-ph.GA]];

[16] S. Floerchinger, N. Tetradis and U. A. Wiedemann, arXiv:1506.00407 [gr-qc], contribution to the MG14 proceedings; D. Blas, S. Floerchinger, M. Garny, N. Tetradis and U. A. Wiedemann, JCAP 1511, 049 (2015) doi:10.1088/1475-7516/2015/11/049 [arXiv:1507.06665 [astro-ph.CO]].

[17] J. F. Navarro, C. S. Frenk, and S. D. M. White, Astrophys. J. 490, 493 (1997) http://adsabs.harvard.edu/abs/1997ApJ...490..493N [astro-ph/9611107]

[18] B. Moore, Nature 370, 629 - 631 (25 August 1994); doi:10.1038/370629a0; W. J. G. de Blok, The Core-Cusp Problem, Advances in Astronomy 2010789293 (2010) [arXiv:0910.3538]; Se-Heon Oh et al., Astrophys. J. 149 (6), 96 (2015).

[19] B. Moore, S. Ghigna, F. Governato, G. Lake, T. R. Quinn, J. Stadel and P. Tozzi, Astrophys. J. 524, L19 (1999) doi:10.1086/312287 [astro-ph/9907411]; A. A. Klypin, A. V. Kravtsov, O. Valenzuela and F. Prada, Astrophys. J. 522, 82 (1999) doi:10.1086/307643 [astro-ph/9901240]; E. Polisensky and M. Ricotti, Phys. Rev. D 83, 043506 (2011) doi:10.1103/PhysRevD.83.043506 [arXiv:1004.1459 [astro-ph.CO]].

[20] M. Boylan-Kolchin, J. S. Bullock and M. Kaplinghat, Mon. Not. Roy. Astron. Soc. 415, L40 (2011) [arXiv:1103.0007]; ibid. 422, 1203 (2012) [arXiv: 1111.2048].

[21] T. Richardson and M. Fairbairn, Mon. Not. Roy. Astron. Soc. 441, no. 2, 1584 (2014) doi:10.1093/mnras/stu691 [arXiv:1401.6195 [astro-ph.GA]].

[22] J.Navarro et al. Mon. Not. Roy. Astron. Soc. 283 L72 (1996) doi:10.1093/mnras/283.3.72L; A. Pontzen et al., Nature 42, 3464 (2012) doi:10.1111/j.1365-2966.2012.20571.

[23] M. Lovell et al., Mon. Not. Roy. Astron. Soc. 420, 2318 (2012). doi:10.1111/j.1365-2966.2011.20200.x O. Elbert et al. Mon. Not. Roy. Astron. Soc. 453, 29 (2015) doi:10.1093/mnras/stv1470

[24] J.S. Bullock, Notes on the Missing Satellites Problem [arXiv:1009.4505], Bibliographic Code: 2010arXiv1009.4505B, and references therein.

[25] J. D. Simon and M. Geha, Astrophys. J. 670, 313 (2007) doi:10.1086/521816 [arXiv:0706.0516 [astro-ph]].

[26] D. N. Spergel and P. J. Steinhardt, Phys. Rev. Lett. 84, 3760 (2000) doi:10.1103/PhysRevLett.84.3760 [astro-ph/9909386].

[27] M. Rocha, A. H. G. Peter, J. S. Bullock, M. Kaplinghat, S. Garrison-Kimmel, J. Onorbe and L. A. Moustakas, Mon. Not. Roy. Astron. Soc. 430, 81 (2013) doi:10.1093/mnras/sts514 [arXiv:1208.3025 [astro-ph.CO]].

[28] S. W. Randall, M. Markevitch, D. Clowe, A. H. Gonzalez and M. Bradac, Astrophys. J. 679, 1173 (2008) doi:10.1086/587859 [arXiv:0704.0261 [astro-ph]]. 
[29] F. Kahlhoefer, K. Schmidt-Hoberg, M. T. Frandsen and S. Sarkar, Mon. Not. Roy. Astron. Soc. 437, no. 3, 2865 (2014) doi:10.1093/mnras/stt2097 [arXiv:1308.3419 [astro-ph.CO]].

[30] F. Kahlhoefer, K. Schmidt-Hoberg, J. Kummer and S. Sarkar, Mon. Not. Roy. Astron. Soc. 452, no. 1, L54 (2015) doi:10.1093/mnrasl/slv088 [arXiv:1504.06576 [astro-ph.CO]].

[31] R. Massey, L.L.R. Williams, R. Smit, M. Swinbank, T. Kitching et al., Mon. Not. Roy. Astron. Soc. 449, 3393 (2015).

[32] H.-Y. Schive, M.-H. Liao, T.-P. Woo, S.-K. Wong, T. Chiueh, T. Broadhurst, and W.-Y. P. Hwang, Phys. Rev. Lett. 113, 261302 (2014) doi:10.1103/PhysRevLett.113.261302 [arXiv:1407.7762]

[33] T. Asaka, S. Blanchet and M. Shaposhnikov, Phys. Lett. B 631, 151 (2005) doi:10.1016/j.physletb.2005.09.070 [hep-ph/0503065];

for reviews see: M. Shaposhnikov, Progress of Theoretical Physics 122, 185 (2009); A. Boyarsky, O. Ruchayskiy and M. Shaposhnikov, Ann. Rev. Nucl. Part. Sci. 59, 191 (2009) doi:10.1146/annurev.nucl.010909.083654 [arXiv:0901.0011 [hep-ph]] and references therein.

[34] K. C. Y. Ng, S. Horiuchi, J. M. Gaskins, M. Smith and R. Preece, Phys. Rev. D 92, no. 4, 043503 (2015) doi:10.1103/PhysRevD.92.043503 [arXiv:1504.04027 [astro-ph.CO]].

[35] C. R. Argüelles, R. Ruffini, and B. M. O. Fraga, J. Kor. Phys. Soc. 65, 809 (2014) doi:10.3938/jkps.65.809 [arXiv:1402.1329]

[36] See, for instance: A. Buonaura [for the SHiP Collaboration], PoS NEUTEL 2015, 064 (2015) and references therein; S. Alekhin et al., "A facility to Search for Hidden Particles at the CERN SPS: the SHiP physics case," arXiv:1504.04855 [hep-ph]. 